CARNETS DE Carnets de géographes

GÉOGRAPHES.

15 | 2021

Les dimensions spatiales du maintien de l'ordre

\title{
BABELS, La police des migrants. Filtrer, disperser, harceler
}

Paris : éditions du Passager clandestin, 2019, 120 p.

\section{Léopoldine Manac'h}

\section{OpenEdition}

Journals

Édition électronique

URL : https://journals.openedition.org/cdg/6966

DOI : $10.4000 /$ cdg. 6966

ISSN : 2107-7266

Éditeur

UMR 245 - CESSMA

Référence électronique

Léopoldine Manac'h, «BABELS, La police des migrants. Filtrer, disperser, harceler », Carnets de géographes [En ligne], 15 | 2021, mis en ligne le 03 avril 2021, consulté le 28 mai 2021. URL : http:// journals.openedition.org/cdg/6966; DOI : https://doi.org/10.4000/cdg.6966

Ce document a été généré automatiquement le 28 mai 2021.

\section{c) (i) $\Theta$}

La revue Carnets de géographes est mise à disposition selon les termes de la Licence Creative Commons Attribution - Pas d'Utilisation Commerciale - Pas de Modification 4.0 International. 


\title{
BABELS, La police des migrants. Filtrer, disperser, harceler
}

Paris : éditions du Passager clandestin, 2019, 120 p.

\author{
Léopoldine Manac'h
}

\section{RÉFÉRENCE}

BABELS, La police des migrants. Filtrer, disperser, harceler, Coordonné par Sarah Barnier, Sara Casella Colombeau, Camille Gardesse, Camille Guenebeaud \& Stefan Le Courant, Paris : Éditions du Passager clandestin, 2019, 120 p.

1 L'ouvrage intitulé La police des migrants. Filtrer, disperser, harceler du collectif de recherche Babels décrit l'omniprésence des forces de police dans la répression des migrations et ses conséquences en terme de violences ${ }^{1}$. Dans la lignée de la collection «Bibliothèque des frontières » des éditions du Passager clandestin signée par Babels, le livre a pour ambition de rendre accessible à un lectorat plus large que le seul public scientifique des clefs de lecture relatives à la crise de l'accueil des exilées. Politique et intellectuelle, la démarche du collectif vise à restituer au sujet sa profondeur historique et analytique à partir d'un croisement entre les causes et les effets de ces politiques migratoires répressives, dont le bras armé est la police.

2 En forgeant le terme de "police des migrants", les auteur·es cherchent à décrire en quoi les techniques et pratiques policières d'entrave à la migration se ressemblent et se perpétuent le long des routes migratoires en «transcendant les contextes géographiques et politiques » (p. 8). Ils proposent d'aborder la question à la fois à partir des répertoires d'action policiers et des expériences faites par les personnes migrantes de la confrontation avec les forces de police. Le but de ce travail collectif est ainsi de rendre visible et intelligible les violences policières à l'encontre des personnes migrantes comme des stratégies de gouvernement des migrations.

3 L'expression de "police des migrants » engage de plus dans une lutte discursive au fondement du projet d'écriture du livre par la contestation des prismes majoritaires 
d'analyse en terme d'insécurité et de risque migratoire ${ }^{2}$. Les auteur'es remarquent en introduction que l'inflation langagière du terme "migrant" révèle "les nouvelles modalités de l'illégalité en refusant de reconnaître que la personnes est arrivée " (p. 15). Iels reviennent ainsi sur la redéfinition idéologique de la migration à partir des années 1970, en procédant à un rappel socio-historique de «l'épaississement de la frontière » de cette décennie, qui voient le déplacement des contrôles vers les espaces frontaliers. Cela s'accompagne d'un changement d'échelle des pratiques policières: le contrôle ne s'effectue plus seulement sur le territoire mais par des entraves aux déplacements en amont.

Dans cet ouvrage foisonnant à l'argumentation très serrée, la multiplication des terrains d'enquête permet de croiser les analyses depuis les côtes de la Méditerranée jusqu'aux rues de Paris et Calais en passant par des zones de frontières telles que les enclaves espagnoles de Ceuta et Melilla. Cette multiplicité se retrouve dans la diversité des voix que le livre permet de croiser ; le dialogue auquel il ouvre entre chercheurses en sciences sociales, personnes migrantes, et militantes et bénévoles des droits des migrantes permet de restituer avec finesse la densité de l'expérience de la confrontation avec la police.

Les répertoires d'actions policières sont catégorisées selon trois types qui composent la structure argumentative du livre : le filtrage, la dispersion et le harcèlement.

\section{Filtrer, diviser}

6 À partir de la définition de la frontière comme lieu du filtrage, la première partie est consacrée à l'analyse «des pratiques policières de ciblage des personnes migrantes et leurs effets»(p. 21). Les auteur.es décryptent les circulations et les réemplois des techniques d'identification et de refoulement des migrantes entre différents États. Le travail quotidien des policieres aux frontières est relié à l'analyse de "l'importation des instruments et des discours européens de lutte contre l'immigration illégale.» (p. 33) qui structure une idéologie sécuritaire commune sur tout le trajet migratoire. Un bref retour sur les contrôles au faciès est fait dans le but de rappeler que les frontières sont aussi internes aux États et incarnées par les agentes de police. Les arrestations et expulsions n'ont pas seulement pour but de réduire la présence de personnes migrantes, tant «il s'agit également d'imposer un ordre social» (p. 50), fruit d'une histoire coloniale dont la police des migrants renforce les structures en la réactivant quotidiennement.

\section{Disperser, expulser}

Le second chapitre articule deux acceptions de la dispersion : celle de la responsabilité du contrôle parmi les acteurs de la société civile et celle de la dispersion des personnes migrantes comme stratégie policière. La délégation du contrôle se fait ainsi par la prévalence des logiques sécuritaires dans les espaces du travail, du soin, de l'éducation, de l'accès aux aides sociales. "Les personnes migrantes voient se restreindre drastiquement les espaces à l'abri des frontières, toutes ces institutions rejouant les opérations de tri et de mise à l'écart qui y prévalent» (p. 56), ce qui contribue à la fragilisation de leurs conditions de vie. Cette délégation se fait en outre à des acteurs privés, notamment dans les technologies de surveillance frontalières (Rodier, 2002) ou 
dans l'obligation de la coopération des acteurs privés tels que les transporteurs maritimes. La dispersion des personnes migrantes est analysée à l'échelle interétatiques (pratiques de refoulements aux frontières), étatique (déplacements forcés loin d'une frontière comme à Calais, maillages de dispositifs d'hébergement sur l'ensemble d'un territoire) mais aussi locale, avec l'exemple des populations rroms expulsées des territoires municipaux.

\section{Harceler, décourager}

8 Les auteures rentrent enfin dans l'épaisseur des effets de ces violences, aux plans corporels et psychologiques. À partir des expériences de la banalisation de violences exercées par des agentes de l'État et de ses conséquences sur la santé mentale des personnes migrantes, ils s'emploient à décrypter un "gouvernement par l'insécurisation » (p. 85). Il est montré que ces pratiques dissuasives n'ont pas l'effet escompté (le départ des migrantes) mais leur rendent en revanche la vie insupportable. La catégorie de torture est en ce sens convoquée par des psychologues à Calais afin de décrire le caractère systématique des faits de violences policières, leur multiplicité et la déshumanisation qu'ils produisent (p. 94-99). Les brutalités policières sont en outre analysées à travers leurs effets sur l'engagement dans les mobilisations pour les migrantes et leurs soutiens. L'analyse des expulsions de campements parisiens montre ainsi que la confrontation directe ou indirecte avec des faits de violence (coups de matraque, gaz lacrymogènes, arrestations) ont précipité ou découragé l'investissement de soutiens.

\section{All Cops Are Borders. Spatialisation de la violence policière}

9 Cet ouvrage permet d'envisager la dimension spatiale du gouvernement policier des migrations tant aux frontières nationales que dans les façons dont la menace de la confrontation policière dans les rues des villes ploie sur les déplacements des personnes étrangères qui développent des stratégies d'évitement. Il donne en outre l'occasion de rentrer dans la compréhension de la manière dont se multiplient et s'articulent des frontières (physiques, sociales, raciales et symboliques) auxquelles les personnes migrantes se heurtent et qu'elles traversent quotidiennement. Les aspects raciaux et genrés de la répression sont à plusieurs reprises évoqués en tant qu'ils réactivent ces types de hiérarchisations ancrées historiquement ${ }^{3}$. Le prisme de lecture par les répertoires d'actions et leurs effets offre par ailleurs la possibilité au collectif Babels de décentrer l'analyse des pratiques d'une seule police des migrants pour ouvrir à une ressaisie plus large du continuum de violences policières qui frappent les personnes racialisées, plus spécifiquement issues des quartiers populaires.

\section{Normalisation de la répression et découragement}

10 Le gouvernement policier des migrations est légitimé par un arsenal idéologique, législatif et médiatique de constitution des migrantes en dangers. Ainsi, la criminalisation des personnes étrangères ou racialisées justifie le raffinement des 
modes de contrôle et de répression. Cette technicisation et cette standardisation de la police des migrants participent à sa dépolitisation et à sa normalisation. Pour conclure, je vais revenir sur la compréhension des effets de cette normalisation d'un état de fait policier et de ses effets sur les subjectivités et sur les mobilisations politiques.

Le livre montre très clairement le resserrement du maillage policier sur toutes les facettes de la vie des personnes étrangères pour qui les horizons se bouchent. Les confrontations quotidiennes avec la violence légitime et impunie des forces de l'ordre, les restrictions des accès aux droits qui infusent des logiques sécuritaires dans tous les pans de la société, les échecs des mobilisations et leur répression violente découragent les personnes étrangères et leurs soutiens, cibles indirectes de cette violence. En prenant à bras le corps les conséquences psychiques, individuelles et collectives, du découragement, on pourrait dès lors faire l'hypothèse qu'il s'agit d'une stratégie de gouvernement, voire d'une "gouvernementalité du découragement " (Manac'h, 2019) dont la compréhension permettrait peut-être de construire les outils politiques et théoriques de sa contestation.

Cette articulation entre répression et dépolitisation n'est par ailleurs pas l'apanage des politiques migratoires. Les auteures rappellent en ouverture que cette administration policière d'un fait social s'inscrit dans une tendance plus large de gouvernement: les réponses aux enjeux sociaux, politiques et économiques contemporains sont confiées exclusivement aux forces de police. Cela conduit à des explosions de violences policières très rarement sanctionnées et légitimées par ce que la politologue Vanessa Codaccioni désigne comme une "criminalisation dépolitisante de l'activisme oppositionnel » (2019: 13), notamment lors de manifestations ou d'occupations. On pense ici à la répression qui a suivi l'occupation pacifique du Panthéon par le mouvement des Gilets Noirs le 12 juillet 20194. L'expérience commune de cette violence, par un contrôle au faciès humiliant ou une charge policière lors d'un samedi de manifestation Gilets Jaunes façonne un " horizon politique » collectif (Gould 2009) qui conteste radicalement le fait « que la police a toujours bien fait son travail $»^{5}$.

\section{BIBLIOGRAPHIE}

CHAMAYOU G. (2010), Les chasses à l'homme, histoire et philosophie du pouvoir cynégétique, Paris, La Fabrique.

CODACCIONI V. (2019), Répression. L'État face aux contestations politiques, Paris, Textuel.

EINAUDI J-L. (2007), La bataille de Paris. 17 octobre 1961, Paris, Seuil.

FASSIN D. (2011), La force de l'ordre. Une anthropologie de la police des quartiers, Paris, Seuil.

GOULD D. (2009), Moving Politics. Emotion and ACT UP's Fight Against AIDS, Chicago, University of Chicago Press.

JOBARD F. (2015), « La police en banlieue après les émeutes de 2005 », Mouvements, vol. 83, no. 3, pp. 75-86. 
MANAC'H L. (2019), « Faire face aux politiques de découragement », Savoirs des luttes, luttes des savoirs. En ligne : https://sdl.hypotheses.org/3072

RIGOUSTE M. (2012), La domination policiere. Une violence industrielle, Paris, La Fabrique.

RODIER C. (2012), Xénophobie Business. À quoi servent les contrôles migratoires ?, Paris, La Découverte.

\section{NOTES}

1. Le collectif Babels (Borders Analysis and Borders Ethnographies in Liminal Situations) est issu d'un programme financé par l'ANR coordonné par Michel Agier et Stefan Le Courant entre 2016 et 2019. Il a eu pour vocation de rassembler des chercheurses proposant des ethnographies de situations de frontière contemporaines dans des villes européennes et méditerranéennes. Voir les autres titres de la collection tels que De Lesbos à Calais : comment l'Europe fabrique des camps (2017) ou Entre accueil et rejet : ce que les villes font aux migrants (2018).

2. Je fais notamment référence au terme forgé par E. Macron d'« insécurité culturelle » alors que le gouvernement définit la migration comme priorité pour les Français·es.

3. De nombreux'ses auteur'es s'attachent à décrire la racialisation du maintien de l'ordre (Fassin, 2011 ; Rigouste, 2012 ; Jobard, 2015) et les façons dont elle plonge ses racines dans son histoire coloniale (Einaudi, 2007 ; Chamayou, 2010)

4. Voir les vidéos tournées par les journalistes du média Basta dans l'article de Thomas Clerget, "Occupation du Panthéon: "Ils ont voulu terroriser des gens qui ont relevé la tête, et qui n'ont plus peur" ", Basta, 15 juillet 2019 [en ligne] https://www.bastamag.net/Pantheon-gilets-noirsoccupation-sans-papiers-mal-loges-Republique-repression-violences-policieres (consulté le 16 juillet 2019).

5. Diam's. « Ma France à moi », Dans ma bulle, Tefa/Masta, 2006.

\section{AUTEURS}

\section{LÉOPOLDINE MANAC'H}

Doctorante en anthropologie au Ceped (Centre Population et Développement), université Paris Descartes.

leopoldine.manach[at]gmail.com 